\title{
Semantic Memory Association, Procedural Grammar Syntax and Episodic Modality Coordination as Three Interactive Neural Processes Organizing Language: A Model
}

\author{
Zi-Jian Cai \\ Suzhou, China \\ Email: $\underline{\text { hrsh8@126.com }}$
}

Received 30 June 2015; accepted 15 July 2015; published 21 July 2015

Copyright (C) 2015 by author and OALib.

This work is licensed under the Creative Commons Attribution International License (CC BY). http://creativecommons.org/licenses/by/4.0/

c) (†) Open Access

\begin{abstract}
In the declarative/procedural model of language, it assumes the semantic words as declarative memories while the grammatical syntax as procedural rules, whereas it is herein suggested that different words associate with different cortical modalities, so that it is necessary to consider the modality coordination of words episodic in meanings in sentences. In evidence, stuttering is intact on grammar but impaired on fluency, so that the dopaminergic system, with antagonists alleviating and genes phenotyping stuttering, may involve linguistic modality disorganization. Reversely, the gamma band correlates with word congruency, so that the cholinergic projections may help cortical modality coordination. There are present discourse deficits, so that story narration also requires cortical modality coordination. In these respects, semantic memory association, procedural grammar syntax and episodic modality coordination interact to organize language from word to sentence to story, so that it herein extends the present declarative/procedural model underlying two kinds of neural processes to semantic/syntactic/episodic model of three kinds of neural processes for depiction and simulation of language.
\end{abstract}

\section{Keywords}

Semantic Memory, Procedural Grammar Syntax, Episodic Coordination, Stutter, Dopamine, Gamma Band, Computer Simulation

Subject Areas: Artificial Intelligence, Linguistics, Neuroscience

\section{Introduction}

Language is the high and complex mental activity of humans for communication and meditation. The native

How to cite this paper: Cai, Z.-J. (2015) Semantic Memory Association, Procedural Grammar Syntax and Episodic Modality Coordination as Three Interactive Neural Processes Organizing Language: A Model. Open Access Library Journal, 2: e1718. 
language in adults is organized from word to sentence to story, with linguistic components manifesting variation in meanings and diversity in combinations, making the language able to contain and deliver almost all kinds of information and intention at ease.

Corresponding to the variations and complexities of language components, the symptoms of language disorders, such as aphasia, stutter and discourse disorders, are also diverse and various. There are many kinds of aphasias, such as anomic aphasia, conduction aphasia, expressive aphasia, and their subtypes. Stutter is different from aphasia in that it is intact on words and grammar but impaired on fluency. At higher level, narrative discourse disorders affect story narration rather than individual sentences. Although the linguistic impairments are important in conveying the information about the underlying neural processes of language, the diversities and complexities of the disorders make it difficult to formulate a general neural model to depict and simulate language.

In this article, through insightful reviewing the comprehensive processes of language from word to sentence to story, it attempts to propose a new neural model for the convenience to depict and simulate the linguistic processes.

\section{The Declarative/Procedural Model of Language}

\subsection{Word Comprehension as Memory Associations}

Language is acquired by postnatal learning and memory. Memory can be divided into declarative memory for facts or episodes, and procedural memory for skills or habits. Declarative memory and procedural memory manifest as two kinds of memories different in underlying neural processes. Declarative memory can be divided into short-term memory and long-term memory. Short-term memory is the memory of precedent episodes or facts within minutes or seconds, while long-term memory is the memory of them more than hours or days ago. Besides, remote (retrograde) memory is the long term memory lasting for decades of years. This classification of memories has been illustrated early in numerous reviews [1] and books [2] for many years.

There have been many theories trying to account for the consolidation and retrieval of declarative memory. Notably, one category of theories [2] [3] suggested that the hippocampus and amygdala mediate memory consolidation and retrieval via their direct efferents sent back to the cortex [4]. Another theory, derived from the disconnection theory of amnesia [5] [6], suggested the memory storage site in temporal lobe be connected to the complex cognition site in prefrontal cortex through the thalamus with lesions to which there caused diencephalic amnesia. As the third category, Mishkin and Appenzeller [7] added the cholinergic (ACh) system in basal forebrain to these forebrain memory sites, partly accomplishing the memory functions of them via biochemical consolidation of memory traces in cortex with ACh. In parallel, in 1990 I published the limbic-reticular coupling theory [1], suggesting that the hippocampus and amygdala control and regulate such descending limbic structures as the mammillary bodies, septum, hypothalamus and epithalamus, and in turn regulate the four reticular neuromodulatory systems of noradrenaline (NA), serotonin (5-HT), dopamine (DA) and acetylcholine (ACh), and therefore accomplish declarative memory consolidation and retrieval.

Words in native language are acquired as declarative memories during childhood, so that they are utilized as stable remote (retrograde) memory in adults. Regardless how to transform the new words acquired in childhood into the remote memory of words utilized in adults, the words in native language are the outcomes of the lengthy consolidation processes of declarative memories, as proposed by Pinker, Ullman and so on in their declarative/ procedural model for linguistic processing [8]-[11]. There might be present several mechanisms for the lengthy processes of linguistic consolidation and modification, such as derivation, analogy, categorization and so on [12] [13].

All words have one or several defined specific meanings, so that the words in native language are actually the remote memories in association with one or several specific meanings [8]-[11], which indicates that the words are actually the associative memories as one sort of remote declarative memories.

Evidence in support of this assertion is rich in many reviews [8]-[11]. More typical in consistency in addition, with aphasia as a common sign in many stroke, it was reported in patients with variations in locations of stroke to produce many specific subtypes of aphasias, including anomic aphasia and transcortical sensory aphasia [14]. Further, alexia is characterized as dysfunction in word comprehension during reading and lesion in fusiform gyrus or nearby cortical regions [15]-[17], whereas anomic aphasia is characterized as dysfunction in appropriate naming and lesion in anterior temporal lobe [18]-[20]. Obviously, word comprehension is stored as remote 
memory associations specific in cortical modality.

It is noticed that some derivatives of words are formed from linguistic rules instead of learned from teaching [8] [9]. Nonetheless, word derivatives are also associated with original words or specific meanings, so that they are also present as memory associations in cortices. In this regard, the linguistic rules for forming word derivatives do not affect the conclusion that word comprehension is stored as remote memory associations.

\subsection{Grammar Syntax as Procedural Memory to Associate Words into Sentence}

Pinker, Ullman and so on integrated a declarative/procedural model from two kinds of corresponding neural processes for language. They suggested that, while the mental lexicon of word-specific knowledge depended on remote declarative memory as has just been demonstrated above, the mental grammar of syntactic knowledge would depend on procedural memory subserving the sequential combination of lexical items by procedural rules into sentences [8]-[11]. This is an important advance in understanding the differential neural substrates of lexicon and grammar.

Evidence in support of grammar as procedural memory is also rich in the relevant reviews [8]-[11]. More typical in consistency in addition, there are present a subtype of patients called as non-fluent/agrammatic aphasia who are specially poor in grammatical comprehension and expression [21]. In pathology, the non-fluent/ agrammatic aphasia is associated with frontotemporal degeneration from tauopathy [21], different from the semantic aphasia with frontotemporal degeneration from TDP-43 proteinopathy, and the logopenic aphasia with Alzheimer pathology [21] [22]. Besides, it was also demonstrated that the agrammatic aphasia could be distinguished from the logopenic aphasia with detailed diagnosis [23] [24], such as the Parkinsonian motor features [23]. Further, it was more importantly reported that some agrammatic aphasic patients were simultaneously impaired on artificial grammar learning as compared to controls [25], indicating the breakdown of language in agrammatic aphasia to be associated with damage to those neural structures concurrently subserving both native language and artificial procedural learning of sequence.

It is interesting to note that animals are also able to use finite-state grammars in vocal communications [26]. It is plausible that the grammar and words shared the similar mechanisms in their evolutionary origins during communication.

\section{A New Semantic/Syntactic/Episodic Model of Language to Extend the Declarative/Procedural Model}

The declarative/procedural neural model of language only concerns how the words as associative memories are sequentially associated to form the sentence according to the grammatical syntax as procedural memories. However, herein in this article, it is differently concerned the varieties in meanings of sentences generated from the various words.

If the words of a sentence belong to the same category in semantic classification, the sentence would also fall into the same category in cortical modality. Short term recall of digits recollects all digital words in the same numerical modality of cortex. It was demonstrated that short-term recall of visually presented additive and nonadditive digital materials were performed similarly in deaf and normal subjects [27], indicating modality specificity in cortical processing. In this situation, the digital sentence manifested the same in visual cortical modality as individual digits without affected by the auditory cortex.

If the words of a sentence belong to different categories in semantic classification, especially in sentence with many adjectives and adverbs, the words would represent the specific or episodic association of their meanings in the sentence, even though they follow the procedural rules of grammar syntax to form sentence. Specific or episodic coordination among these different cortical modalities world be necessary for completing the sentence. It was reported that short-term memory treatment improved the ability of aphasia in sentence comprehension as well as in forward digit span, the latter presumably from frontal inhibition of proactive interference, but not in backward digit span, phonological processing and single word comprehension [28], implicating short-term memory treatment improved the patients more on episodic modality coordination than on remote semantic memories, dissociating the two processes in sentence. As another example, older participants were reported superior in memory performance in sentence than younger although both showed similar performance in list [29], demonstrating that the sentence differed from words due to episodic word association in meanings.

Now that the episodic modality coordination of cortices participates in organizing the language in addition to 
the declarative/procedural neural model, then the semantic memory association, procedural grammar syntax and episodic modality coordination interact to organize the neural processes of native language. This new semantic/syntactic/episodic model extends the present declarative/procedural model of language.

\section{Supporting Evidence}

As the declarative/procedural neural model has been extensively reviewed and supported [8]-[11], to prove the new semantic/syntactic/episodic model, it is the key to support the episodic coordination of cortical modalities for language. Evidence in support of episodic coordination of cortical modalities for language is rich in various aspects, such as pharmacological treatment of stuttering, assistant therapy of aphasia, linguistic gamma bands, linguistic acquisition in bilinguals, and so on. In the following discussions, it is inspected on these collections of evidence.

Stuttering is characterized as intact on grammar but impaired on fluency of speaking. Brain imaging studies suggested that stutters manifest alteration in linguistic information processing in various brain regions [30] [31]. More interestingly, the inferior frontal gyrus in the counterpart right hemisphere has been reported as putative compensatory locus unique in stuttering [32] [33], demonstrating change in hemispheric laterality. On the other hand, some therapeutic procedures for treatment of stuttering adopt manipulations across cortical modalities, such as novel speech patterns [34] and altered auditory feedback [35]. Obviously, stuttering patients represent malfunction in episodic coordination of cortical linguistic modalities.

Some stutters can be alleviated with pharmacological interventions. Especially, dopamine antagonists have been shown sometimes to improve the symptoms of stuttering [36] [37], while dopamine agonists to induce stuttering [38]. In further, it was even reported that the dopaminergic genes were associated with stuttering [39]. In this regard, it is obvious that the dopaminergic system is involved in episodic disorganization of cortical linguistic modalities in stutters.

In parallel, aphasic patients receive extensive treatments recently. With concurrent drug therapy in assistance to language therapy, it has been shown that drugs in many therapies act on cholinergic, noradrenergic and glutamatergic neurotransmitters [40] [41], presumably involving the ascending cholinergic system in basal forebrain, ascending noradrenergic projections from Locus Coeruleus and nonspecific activating projections from intralaminar thalamic nuclei. Since the ascending dopaminergic, cholinergic and noradrenergic systems have been demonstrated to be involved in declarative memory consolidation and episodic memory retrieval [1], it would be rational for these systems to participate in episodic coordination of cortical modalities in linguistic processing.

Recently, it has been repeatedly demonstrated that the gamma bands of high frequency associate with the word congruency at sentential level [42]-[44]. As gamma bands are subject to modulation by the ascending cholinergic systems [45] [46], it is obvious that the cholinergic systems are related to the episodic coordination of cortical modalities in sentence processing. Interestingly, compared to the dopaminergic agonists in induction of stuttering [38], the cholinergic systems nonetheless play the opposite roles as manifesting word congruency in sentence.

It is necessary to mention that bilinguals who acquired both languages before 6 years old at age manifest bilateral hemispheric involvement for both languages, whereas those who acquired their second language after 6 years old manifest left hemisphere dominance for both languages [47]. These characteristics of bilinguals indicate that there must have been difference in episodic coordination of cortical modalities for these two types of bilinguals during their ontogenetic development.

Now that it is well evidenced for episodic coordination of cortical modalities at sentential level, it is plausible to in further consider it for story narration. Complex stories certainly require more episodic coordination of cortical modalities. Language disorders occur in narrative discourse skills in patients with various pathologies [48]-[50], demonstrating diversity in cortical modalities. Besides, stuttering is also a disorder of discourse sometimes, as narrative therapy is auxiliary to treatment of stuttering [51] [52]. These facts implicate that episodic modality coordination of cortices is also required at high level during story narration.

It has been suggested that the impairment in completing complex language result from disruption of frontal attention processes [53]. Many authors suggest a rostro-caudal gradient for abstract processing of complex sequence in the frontal cortex [54] [55]. Herein, it is necessary to point out that the frontal function of attention is also exactly one kind of sustained coordination of various cortical modalities, including the rostro-caudal gra- 
dient of frontal cortex [54] [55], which is in further modulated with the ascending cholinergic [56] and dopaminergic systems [57], as well as projections from intralaminar thalamic nuclei [58].

In all, it is herein demonstrated that, in addition to the declarative/procedural model underlying two kinds of neural processes, the episodic modality coordination of cortices is also well evidenced as the third kind of neural processes to organize language, as followings: 1 ) The dopaminergic system may be involved in the linguistic modality disorganization, as the dopaminergic antagonists alleviate and genes phenotype stuttering. 2) From drugs in many assisting therapies of aphasia, it is implicated that the ascending cholinergic system, noradrenergic projections and nonspecific activating projections from intralaminar thalamic nuclei may participate in episodic coordination of cortical modalities in linguistic processing. 3) It is evidenced that the gamma bands of high frequency, subject to modulation by the ascending cholinergic systems, associate with the word congruency at sentential level and the episodic coordination of cortical modalities. 4) The characteristics in ontogenetic development of bilinguals indicate that there must have been difference in episodic coordination of cortical modalities for the two types of bilinguals acquiring before and after 6 years old at age. 5) Complex stories would require more for episodic cortical modality coordination than individual sentences.

In summary, semantic memory association, procedural grammar syntax and episodic modality coordination interact to organize the native language from word to sentence to story, which is exactly the semantic/syntactic/ episodic model of three kinds of neural processes extending the present declarative/procedural model of two kinds of neural processes for language.

\section{Discussions}

The semantic/syntactic/episodic model of language takes over the advantage of the present declarative/procedural model as intuitive relating to the underlying neural processes. One the one hand, the working memory has been addressed by some authors in consideration of memory and language [59]. In neurobiology, working memory is performed with the complicated processes of frontal rostro-caudal gradient of sequence in combination with coordination of various posterior cortical modalities. It could be helpful to supplement some additional memory processes to the declarative/procedural model from working memory, but would be difficult to derive out a new neural model as well organized as the declarative/procedural model. On the other hand, in this article, the adopted episodic coordination of cortical modalities is intuitively revealed as modulation with the ascending dopaminergic, cholinergic and noradrenergic systems which are deficient in the present declarative/procedural model. Semantic memory association, procedural grammar syntax and episodic modality coordination manifest as three interactive neural processes to depict the native language from word to sentence to story. It is the adoption of episodic coordination of cortical modalities that extends the present declarative/procedural model of two kinds of neural processes to a new semantic/syntactic/episodic model of three kinds of neural processes.

There are still some other phenomena likely favorable to the semantic/syntactic/episodic model of language instead of the present declarative/procedural model, which deserves to mention for future direction of research. It is interesting to note that the rapid eye movement (REM) dream sleep is responsible for retention of emotional memories acquired during waking [60]-[62], with cease in firing of noradrenergic and serotonergic systems, but maintenance in activity of cholinergic and dopaminergic systems [60]-[62]. Delusional contents in dreams, such as flying lions and golden houses, are within the scientific scope of investigation in modern psychoanalysis [63] [64]. The dream delusions indicate that the coordination of cortical modalities is disorganized during dream. In this regard, the episodic linguistic processes may also be disturbed during REM sleep, especially the lengthy and complex planning or procedural narrations. Besides, it is still short of results on the linguistic performance of stutters and narrative disorders during dream. All these issues deserve further investigations.

\section{Brief Perspectives}

The present declarative/procedural linguistic model of two kinds of neural processes is famous and influential due to two advantages as followings: 1) It provides an intuitive and simple neural model for the convenience to depict the complex linguistic neural processes. 2) It provides an intuitive and simple neural model for the convenience to simulate the complex linguistic neural processes in computers and robots.

Since the new semantic/syntactic/episodic linguistic model of three kinds of neural processes proposed herein extends and improves the present declarative/procedural model of two kinds of neural processes, it also extends and improves the two advantages of the present declarative/procedural model prospectively, as followings: 1) It 
provides an intuitive but improved neural model for the depiction of the complex linguistic neural processes. 2) It provides an intuitive but improved neural model for simulation of the complex linguistic neural processes for computers and robots. In simulation, it is easy and simple to substitute the two constituents in declarative/procedural model with the three constituents in semantic/syntactic/episodic model.

\section{Conclusion}

In this article, following the recent declarative/procedural neural model of Pinker, Ullman and so on, it is concisely reviewed that word comprehension is stored as remote memory associations in native language, while grammar is actually the procedural memory of linguistic syntax to associate words sequentially to form sentence in accordance. Nonetheless, it is newly suggested in this article that different words in sentence associate with different cortical modalities, so that it is necessary to consider the episodic modality coordination for different outcomes in meanings of sentences. It is pointed out that, as the dopaminergic antagonists alleviate and genes phenotype stuttering, the dopaminergic system may be involved in the linguistic modality disorganization. Besides, since concurrent drug therapy as assistance to language therapy helps to improve the treatments for aphasic patients, other ascending projections such as the cholinergic, noradrenergic and glutamatergic systems may also be involved in the episodic cortical modality coordination. Again, the cholinergic gamma bands have been repeatedly reported to associate with the word congruency at sentential level, related to episodic cortical modality coordination. Further, complex stories certainly require more for episodic cortical modality coordination. Whilst, episodic coordination of cortical modalities is more advantageous than working memory in homogeneity of underlying neural processes, simply as cortical modulation from the ascending nonspecific projections which are deficient in the present declarative/procedural neural model. In these respects, semantic memory association, procedural grammar syntax and episodic modality coordination interact to organize the native language from word to sentence to story. This is a new semantic/syntactic/episodic model of three kinds of neural processes extending the present declarative/procedural model of two kinds of neural processes to depict and simulate the complex processes of language.

\section{Conflict of Interest}

The author declares no conflict of interest or financial support for this work.

\section{Acknowledgements}

The author would like to express his gratitude to MingXun Cai for his granting to pay for the Open Access charge of this paper.

\section{References}

[1] Cai, Z.J. (1990) The Neural Mechanism of Declarative Memory Consolidation and Retrieval: A Hypothesis. Neuroscience \& Biobehavioral Reviews, 14, 295-304. http://dx.doi.org/10.1016/S0149-7634(05)80039-9

[2] Squire, L.R. (1987) Memory and Brain. Oxford University Press, Oxford.

[3] Teyler, T.J. and DiScenna, P. (1985) The Role of Hippocampus in Memory: A Hypothesis. Neuroscience \& Biobehavioral Reviews, 9, 377-389. http://dx.doi.org/10.1016/0149-7634(85)90016-8

[4] Saunders, R.C. and Rosene, D.L. (1988) A Comparison of the Efferents of the Amygdala and the Hippocampal Formation in the Rhesus Monkey: I. Convergence in the Entorhinal, Prorhinal and Perirhinal Cortices. Journal of Comparative Neurology, 271, 153-184. http://dx.doi.org/10.1002/cne.902710202

[5] Mishkin, M. (1982) A Memory System in the Monkey. Philosophical Transactions of the Royal Society of London. Series B, Biological Sciences, 298, 85-95. http://dx.doi.org/10.1098/rstb.1982.0074

[6] Warrington, E.K. and Weiskrantz, L. (1982) Amnesia: A Disconnection Syndrome? Neuropsychologia, 20, $233-248$. http://dx.doi.org/10.1016/0028-3932(82)90099-9

[7] Mishkin, M. and Appenzeller, T. (1987) The Anatomy of Memory. Scientific American, 256, 80-89. http://dx.doi.org/10.1038/scientificamerican0687-80

[8] Pinker, S. (1991) Rules of Language. Science, 253, 530-535. http://dx.doi.org/10.1126/science.1857983

[9] Ullman, M.T., Corkin, S., Coppola, M., Hickok, G., Growdon, J.H., Koroshetz, W.J. and Pinker, S. (1997) A Neural Dissociation within Language: Evidence That the Mental Dictionary Is Part of Declarative Memory, and That Gram- 
matical Rules Are Processed by the Procedural System. Journal of Cognitive Neuroscience, 9, 266-276. “ http://dx.doi.org/10.1162/jocn.1997.9.2.266

[10] Ullman, M.T. (2004) Contributions of Memory Circuits to Language: The Declarative/Procedural Model. Cognition, 92, 231-270. http://dx.doi.org/10.1016/j.cognition.2003.10.008

[11] Ardila, A. (2012) Interaction between Lexical and Grammatical Language Systems in the Brain. Physics of Life Reviews, 9, 198-214. http://dx.doi.org/10.1016/j.plrev.2012.05.001

[12] Lerner, I. and Shriki, O. (2014) Internally- and Externally-Driven Network Transitions as a Basis for Automatic and Strategic Processes in Semantic Priming: Theory and Experimental Validation. Frontiers in Psychology, 5, 314. http://dx.doi.org/10.3389/fpsyg.2014.00314

[13] Tamminen, J., Davis, M.H. and Rastle, K. (2015) From Specific Examples to General Knowledge in Language Learning. Cognitive Psychology, 79, 1-39. http://dx.doi.org/10.1016/j.cogpsych.2015.03.003

[14] Yang, Z.H., Zhao, X.Q., Wang, C.X., Chen, H.Y. and Zhang, Y.M. (2008) Neuroanatomic Correlation of the PostStroke Aphasias Studied with Imaging. Neurological Research, 30, 356-360. http://dx.doi.org/10.1179/174313208X300332

[15] Turkeltaub, P.E., Goldberg, E.M., Postman-Caucheteux, W.A., Palovcak, M., Quinn, C., Cantor, C. and Coslett, H.B. (2014) Alexia Due to Ischemic Stroke of the Visual Word form Area. Neurocase, 20, 230-235. http://dx.doi.org/10.1080/13554794.2013.770873

[16] Leśniak, M., Soluch, P., Stępień, U., Czepiel, W. and Seniów, J. (2014) Pure Alexia after Damage to the Right Fusiform Gyrus in a Right-Handed Male. Neurologia i Neurochirurgia Polska, 48, 373-377. http://dx.doi.org/10.1016/j.pjnns.2014.09.003

[17] Kleinschmidt, A. and Cohen, L. (2006) The Neural Bases of Prosopagnosia and Pure Alexia: Recent Insights from Functional Neuroimaging. Current Opinion in Neurology, 19, 386-391. http://dx.doi.org/10.1097/01.wco.0000236619.89710.ee

[18] Hanley, J.R. (2014) Accessing Stored Knowledge of Familiar People from Faces, Names and Voices: A Review. Frontiers in Bioscience, 6, 198-207. http://dx.doi.org/10.2741/E702

[19] Ives-Deliperi, V.L. and Butler, J.T. (2012) Naming Outcomes of Anterior Temporal Lobectomy in Epilepsy Patients: A Systematic Review of the Literature. Epilepsy \& Behavior, 24, 194-198. http://dx.doi.org/10.1016/j.yebeh.2012.04.115

[20] Semenza, C. (2011) Naming with Proper Names: The Left Temporal Pole Theory. Behavioural Neurology, 24, $277-$ 284. http://dx.doi.org/10.1155/2011/650103

[21] Grossman, M. (2012) The Non-Fluent/Agrammatic Variant of Primary Progressive Aphasia. Lancet Neurology, 11, 545-555. http://dx.doi.org/10.1016/S1474-4422(12)70099-6

[22] Mesulam, M.M. (2013) Primary Progressive Aphasia and the Language Network: The 2013 H. Houston Merritt Lecture. Neurology, 81, 456-462. http://dx.doi.org/10.1212/WNL.0b013e31829d87df

[23] Graff-Radford, J., Duffy, J.R., Strand, E.A. and Josephs, K.A. (2012) Parkinsonian Motor Features Distinguish the Agrammatic from Logopenic Variant of Primary Progressive Aphasia. Parkinsonism \& Related Disorders, 18, 890-892. http://dx.doi.org/10.1016/j.parkreldis.2012.04.011

[24] Thompson, C.K., Cho, S., Hsu, C.J., Wieneke, C., Rademaker, A., Weitner, B.B., Mesulam, M.M. and Weintraub, S. (2012) Dissociations between Fluency and Agrammatism in Primary Progressive Aphasia. Aphasiology, 26, 20-43. http://dx.doi.org/10.1080/02687038.2011.584691

[25] Christiansen, M.H., Louise Kelly, M., Shillcock, R.C. and Greenfield, K. (2010) Impaired Artificial Grammar Learning in Agrammatism. Cognition, 116, 382-393. http://dx.doi.org/10.1016/j.cognition.2010.05.015

[26] ten Cate, C. and Okanoya, K. (2012) Revisiting the Syntactic Abilities of Non-Human Animals: Natural Vocalizations and Artificial Grammar Learning. Philosophical Transactions of the Royal Society of London. Series B, Biological Sciences, 367, 1984-1994. http://dx.doi.org/10.1098/rstb.2012.0055

[27] Kool, V.K., Pathak, K.C. and Singh, S. (1983) Short-Term Recall of Visually Presented Additive and Nonadditive Digital Material by Deaf and Hearing Subjects. Journal of General Psychology, 108, 19-25. http://dx.doi.org/10.1080/00221309.1983.9711474

[28] Salis, C. (2012) Short-Term Memory Treatment: Patterns of Learning and Generalisation to Sentence Comprehension in a Person with Aphasia. Neuropsychological Rehabilitation, 22, 428-448. http://dx.doi.org/10.1080/09602011.2012.656460

[29] Matzen, L.E. and Benjamin, A.S. (2013) Older and Wiser: Older Adults’ Episodic Word Memory Benefits from Sentence Study Contexts. Psychology and Aging, 28, 754-767. http://dx.doi.org/10.1037/a0032945

[30] Lu, C., Peng, D., Chen, C., Ning, N., Ding, G., Li, K., Yang, Y. and Lin, C. (2010) Altered Effective Connectivity and Anomalous Anatomy in the Basal Ganglia-Thalamocortical Circuit of Stuttering Speakers. Cortex, 46, 49-67. 
http://dx.doi.org/10.1016/j.cortex.2009.02.017

[31] Liu, J., Wang, Z., Huo, Y., Davidson, S.M., Klahr, K., Herder, C.L., Sikora, C.O. and Peterson, B.S. (2014) A Functional Imaging Study of Self-Regulatory Capacities in Persons Who Stutter. PLoS ONE, 9, e89891. http://dx.doi.org/10.1371/journal.pone.0089891

[32] Preibisch, C., Neumann, K., Raab, P., Euler, H.A., von Gudenberg, A.W., Lanfermann, H. and Giraud, A.L. (2003) Evidence for Compensation for Stuttering by the Right Frontal Operculum. Neuroimage, 20, 1356-1364. http://dx.doi.org/10.1016/S1053-8119(03)00376-8

[33] Etchell, A.C., Johnson, B.W. and Sowman, P.F. (2014) Behavioral and Multimodal Neuroimaging Evidence for a Deficit in Brain Timing Networks in Stuttering: A Hypothesis and Theory. Frontiers in Human Neuroscience, 8, 467. http://dx.doi.org/10.3389/fnhum.2014.00467

[34] Packman, A., Onslow, M. and Menzies, R. (2000) Novel Speech Patterns and the Treatment of Stuttering. Disability and Rehabilitation, 22, 65-79. http://dx.doi.org/10.1080/096382800297132

[35] Lincoln, M., Packman, A. and Onslow, M. (2006) Altered Auditory Feedback and the Treatment of Stuttering: A Review. Journal of Fluency Disorders, 31, 71-89. http://dx.doi.org/10.1016/j.jfludis.2006.04.001

[36] Maguire, G.A., Yu, B.P., Franklin, D.L. and Riley, G.D. (2004) Alleviating Stuttering with Pharmacological Interventions. Expert Opinion on Pharmacotherapy, 5, 1565-1571. http://dx.doi.org/10.1517/14656566.5.7.1565

[37] Stager, S.V., Calis, K., Grothe, D., Bloch, M., Berensen, N.M., Smith, P.J. and Braun, A. (2005) Treatment with Medications Affecting Dopaminergic and Serotonergic Mechanisms: Effects on Fluency and Anxiety in Persons Who Stutter. Journal of Fluency Disorders, 30, 319-335. http://dx.doi.org/10.1016/j.jfludis.2005.09.004

[38] Fetterolf, F. and Marceau, M. (2013) A Case of Bupropion-Induced Stuttering. General Hospital Psychiatry, 35, 574.e7-574.e8. http://dx.doi.org/10.1016/j.genhosppsych.2012.07.003

[39] Lan, J., Song, M., Pan, C., Zhuang, G., Wang, Y., Ma, W., Chu, Q., Lai, Q., Xu, F., Li, Y., Liu, L. and Wang, W. (2009) Association between Dopaminergic Genes (SLC6A3 and DRD2) and Stuttering among Han Chinese. Journal of Human Genetics, 54, 457-460. http://dx.doi.org/10.1038/jhg.2009.60

[40] Small, S.L. and Llano, D.A. (2009) Biological Approaches to Aphasia Treatment. Current Neurology and Neuroscience Reports, 9, 443-450. http://dx.doi.org/10.1007/s11910-009-0066-x

[41] Berthier, M.L., Pulvermüller, F., Dávila, G., Casares, N.G. and Gutiérrez, A. (2011) Drug Therapy of Post-Stroke Aphasia: A Review of Current Evidence. Neuropsychology Review, 21, 302-317. http://dx.doi.org/10.1007/s11065-011-9177-7

[42] Wang, L., Zhu, Z. and Bastiaansen, M. (2012) Integration or Predictability? A Further Specification of the Functional Role of Gamma Oscillations in Language Comprehension. Frontiers in Psychology, 3, 187. http://dx.doi.org/10.3389/fpsyg.2012.00187

[43] Vidal, J.R., Freyermuth, S., Jerbi, K., Hamamé, C.M., Ossandon, T., Bertrand, O., Minotti, L., Kahane, P., Berthoz, A. and Lachaux, J.P. (2012) Long-Distance Amplitude Correlations in the High $\gamma$ Band Reveal Segregation and Integration within the Reading Network. The Journal of Neuroscience, 32, 6421-6434. http://dx.doi.org/10.1523/JNEUROSCI.4363-11.2012

[44] Weiss, S. and Müller, H.M. (2013) The Non-Stop Road from Concrete to Abstract: High Concreteness Causes the Activation of Long-Range Networks. Frontier in Human Neuroscience, 7, 526. http://dx.doi.org/10.3389/fnhum.2013.00526

[45] Cape, E.G., Manns, I.D., Alonso, A., Beaudet, A. and Jones, B.E. (2000) Neurotensin-Induced Bursting of Cholinergic Basal Forebrain Neurons Promotes Gamma and Theta Cortical Activity Together with Waking and Paradoxical Sleep. The Journal of Neuroscience, 20, 8452-8461.

[46] Mena-Segovia, J., Sims, H.M., Magill, P.J. and Bolam, J.P. (2008) Cholinergic Brainstem Neurons Modulate Cortical Gamma Activity during Slow Oscillations. The Journal of Physiology, 586, 2947-2960. http://dx.doi.org/10.1113/jphysiol.2008.153874

[47] Hull, R. and Vaid, J. (2007) Bilingual Language Lateralization: A Meta-Analytic Tale of Two Hemispheres. Neuropsychologia, 45, 1987-2008. http://dx.doi.org/10.1016/j.neuropsychologia.2007.03.002

[48] Miniscalco, C., Hagberg, B., Kadesjö, B., Westerlund, M. and Gillberg, C. (2007) Narrative Skills, Cognitive Profiles and Neuropsychiatric Disorders in 7-8-Year-Old Children with Late Developing Language. International Journal of Language and Communication Disorders, 42, 665-681. http://dx.doi.org/10.1080/13682820601084428

[49] Ash, S., Menaged, A., Olm, C., McMillan, C.T., Boller, A., Irwin, D.J., McCluskey, L., Elman, L. and Grossman, M. (2014) Narrative Discourse Deficits in Amyotrophic Lateral Sclerosis. Neurology, 83, 520-528. http://dx.doi.org/10.1212/WNL.0000000000000670

[50] Youse, K.M. and Coelho, C.A. (2009) Treating Underlying Attention Deficits as a Means for Improving Conversation- 
al Discourse in Individuals with Closed Head Injury: A Preliminary Study. NeuroRehabilitation, 24, 355-364.

[51] DiLollo, A., Neimeyer, R.A. and Manning, W.H. (2002) A Personal Construct Psychology View of Relapse: Indications for a Narrative Therapy Component to Stuttering Treatment. Journal of Fluency Disorders, 27, 19-40. http://dx.doi.org/10.1016/S0094-730X(01)00109-7

[52] Leahy, M.M., O’Dwyer, M. and Ryan, F. (2012) Witnessing Stories: Definitional Ceremonies in Narrative Therapy with Adults Who Stutter. Journal of Fluency Disorders, 37, 234-241. http://dx.doi.org/10.1016/j.jfludis.2012.03.001

[53] Alexander, M.P. (2006) Impairments of Procedures for Implementing Complex Language Are Due to Disruption of Frontal Attention Processes. Journal of the International Neuropsychological Society, 12, 236-247. http://dx.doi.org/10.1017/S1355617706060309

[54] Badre, D. (2008) Cognitive Control, Hierarchy, and the Rostro-Caudal Organization of the Frontal Lobes. Trends in Cognitive Sciences, 12, 193-200. http://dx.doi.org/10.1016/j.tics.2008.02.004

[55] Jeon, H.A. (2014) Hierarchical Processing in the Prefrontal Cortex in a Variety of Cognitive Domains. Frontiers in Systems Neuroscience, 8, 223. http://dx.doi.org/10.3389/fnsys.2014.00223

[56] Avery, M.C., Dutt, N. and Krichmar, J.L. (2014) Mechanisms Underlying the Basal Forebrain Enhancement of TopDown and Bottom-Up Attention. European Journal of Neuroscience, 39, 852-865. http://dx.doi.org/10.1111/ejn.12433

[57] Liemburg, E.J., Knegtering, H., Klein, H.C., Kortekaas, R. and Aleman, A. (2012) Antipsychotic Medication and Prefrontal Cortex Activation: A Review of Neuroimaging Findings. European Neuropsychopharmacology, 22, 387-400. http://dx.doi.org/10.1016/j.euroneuro.2011.12.008

[58] Burk, J.A. and Mair, R.G. (2001) Effects of Intralaminar Thalamic Lesions on Sensory Attention and Motor Intention in the Rat: A Comparison with Lesions Involving Frontal Cortex and Hippocampus. Behavioural Brain Research, 123, 49-63. http://dx.doi.org/10.1016/S0166-4328(01)00202-9

[59] Xu, M., Yang, J., Siok, W.T. and Tan, L.H. (2015) Atypical Lateralization of Phonological Working Memory in Developmental Dyslexia. Journal of Neurolinguistics, 33, 67-77. http://dx.doi.org/10.1016/j.jneuroling.2014.07.004

[60] Cai, Z.J. (1991) The Functions of Sleep: Further Analysis. Physiology \& Behavior, 50, 53-60. http://dx.doi.org/10.1016/0031-9384(91)90497-C

[61] Cai, Z.J. (1995) An Integrative Analysis to Sleep Functions. Behavioural Brain Research, 69, 187-194. http://dx.doi.org/10.1016/0166-4328(95)00005-E

[62] Cai, Z.J. (2015) A New Function of Rapid Eye Movement Sleep: Improvement of Muscular Efficiency. Physiology \& Behavior, 144, 110-115. http://dx.doi.org/10.1016/j.physbeh.2015.03.013

[63] Fischmann, T., Russ, M.O. and Leuzinger-Bohleber, M. (2013) Trauma, Dream, and Psychic Change in Psychoanalyses: A Dialog between Psychoanalysis and the Neurosciences. Frontiers in Human Neuroscience, 7, 877. http://dx.doi.org/10.3389/fnhum.2013.00877

[64] Kiekkas, P., Theodorakopoulou, G., Spyratos, F. and Baltopoulos, G.I. (2010) Psychological Distress and Delusional Memories after Critical Care: A Literature Review. International Nursing Review, 57, 288-296. http://dx.doi.org/10.1111/j.1466-7657.2010.00809.x 